\title{
PROPERTIES OF RELATIVELY-DILUTE PLASMAS IN PULSED-POWER SYSTEMS OBTAINED FROM HIGH-ACCURACY LASER SPECTROSCOPY
}

\author{
K. Tsigutkin ${ }^{\xi}$, E. Stambulchik, R. Doron, D. Osin, V. Bernshtam, Yu. Ralchenko, \\ and Y. Maron \\ Faculty of Physics, Weizmann Institute of Science, \\ Rehovot, 76100, Israel
}

\begin{abstract}
We describe a novel application of laser-spectroscopy to investigate the electric fields and the properties of relatively dilute plasmas under high-power pulses at the nanosecond time scale. The method is based on resonant laser-pumping combined with electron excitations and deexcitations by the plasma electrons, which allows for simultaneous observations of various line intensities (due to both allowed and forbidden transitions). These observations, together with novel line-shape calculations recently developed, allow for obtaining the electron density, electron temperature, and electric fields. Another feature of the method developed is that plasma-doping is incorporated, which, combined with the laser spectroscopy, yields 3D-spatially-resolved (sub-mm) measurements. The study is carried out in a coaxialpulsed-plasma configuration (a Plasma Opening Switch). For the first time, line-shape diagnostics was successfully applied to determine the properties of plasmas with densities down to $10^{13} \mathrm{~cm}^{-3}$ at a sub-microsecond time scale. The electron density was determined rather accurately even though the line profiles were dominated by the instrumental and Doppler broadenings. Such diagnostics can be used for the investigation of highcurrent carrying plasmas formed in High-Power Transmission Lines, Plasma Switches, Ion and Electron Diodes, and Plasma Sources.
\end{abstract}

\section{I.INTRODUCTION}

Atomic physics and spectroscopy are commonly used for reliably diagnosing plasma properties. Plasma diagnostics based on the excitations of quantum transitions in atomic or ionic test species in the plasma using tunable lasers allows for obtaining information on particle densities, temperatures, velocity distributions and their fluctuations down to the kinetic scale $[1,2]$. Also such measurements can be used for studying the electric and magnetic fields in plasmas [3].

For the measurements here discussed we employ the laser-induced-fluorescence (LIF) technique, which is based on a selective excitation of an atomic or ionic transition by a resonant absorption of the laser radiation
[2]. The excited level may then decay radiatively via different transitions, causing a photon emission at various different wavelengths. Due to the laser purmping of excited level, this method provides high sensitivity. Also, since the induced-fluorescence originates from the region along the laser path, viewing the emitted radiation perpendicular to the beam direction allows for measurements with high spatial resolution in $3 \mathrm{D}$. Using a short-pulse excitation laser, the temporal resolution can readily be in the nanosecond-scale. The motivation for this research is the need for high-resolution diagnostics capable of sub-millimeter-scale measurements of the electric fields in the plasma and plasma parameters for pulsed-power conditions. To this end, the LIF technique is combined with plasma-doping arrangements so that the laser is made to excite the dopant species. This allow for reducing considerably the background spontaneous emission (that is large for the plasma constituents) and for using various dopant-species as desired for the various measurements. Also, a novel feature in the present measurements is that the laser-excitation leads to increased intensities of various lines of the excited species, due to collisional excitations and de-excitations of the atomic levels neighboring the laser-pumped level. The resulting rise in the line intensities is highly advantageous since it not only allows for measurable intensity of the forbidden line, but also allows for observations of various lines required for determining both the particle velocities and the electron density and temperature (see below).

Also essential for the investigation of the electric fields and the electron density here described, is the use of recently developed novel line-shape calculations that reliably account for the various contributions to the shapes of both the allowed and forbidden lines [4]. We note that while the impact approximation gives reasonably well the electron contribution to the broadening of the two lines, the quasistatic contribution mainly affects the forbidden-line shape.

\section{II.EXPERIMENTAL SYSTEM}

For this study we use a coaxial-pulsed-plasma configuration commonly used for a Plasma Opening Switch experiments [5].

\footnotetext{
६.email: tsigut@plasma-gate.weizmann.ac.il
} 
In a POS, a large current is driven through prefilled plasma, followed by a fast rise in the plasma resistance and subsequent switching of the current to a parallel load.

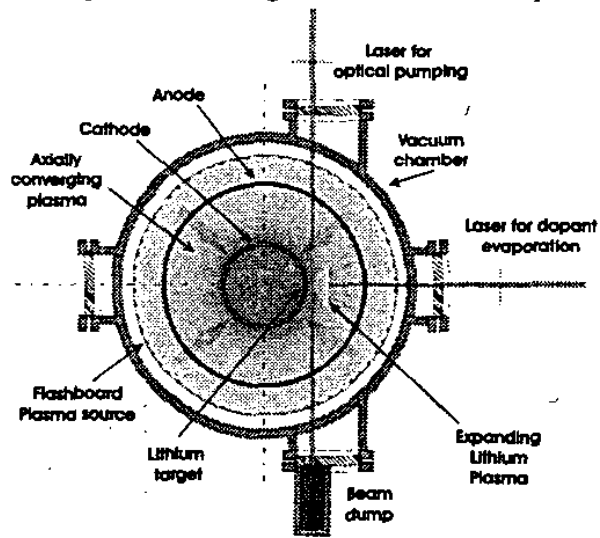

Figure 1. Schematics of the experimental arrangement and diagnostics.

POSs applications can be inductive energy storage, prepulse suppression, and voltage and power multiplication.

As shown in Figure 1, the plasma with $n_{e}=2 \times 10^{j 4}$ $\mathrm{cm}^{-3}$ [6], is produced by a flashboard plasma-source [7] and is injected inwards to prefil the A-K gap. A current pulse of $180 \mathrm{kA}$ with a rise time of $80 \mathrm{~ns}$ is then driven through the plasma. Typical conduction and opening times in this experiment are of 70 and $10 \mathrm{~ns}$, respectively.

In the LIF technique here discussed, the flashboard plasma is doped with a laser-produced lithium beam using a Nd:YAG laser of $5 \times 10^{7} \mathrm{~W} / \mathrm{cm}^{2}$ intensity. A tunable dye laser is then used to pump a selected transition of LiI (see Figure 1). The dye laser, $15 n s$ in duration, delivers a power density of $1 \mathrm{MW} / \mathrm{cm}^{2}$ at the observation region. The spot size of the dye laser, which can be reduced to a sub$\mathrm{mm}$ scale, determines the spatial resolution of the measurements.

The diagnostic system contains two 1-m UV-visible spectrometers. The output of one spectrometer is collected by an optical-fiber array and transmitted to 10 photomultiplier tubes that allow for recording the lineprofile time-dependence, where the output of the other spectrometer is recorded by a gated (down to $5 \mathrm{~ns}$ ) intensified CCD camera, allowing for recording broadband spectra. The spectral resolutions of both systems are $0.1 \AA$.

\section{THE LiI ATOMIC SYSTEM}

Our diagnostic method is based on LIF combined with line-shape analysis of dipole-forbidden transitions [8,9]. The diagnostics makes use of the LiI $2 p-4 d$ (dipoleallowed) and the $2 p-4 f$ (dipole-forbidden) transitions. The dye laser is tuned to excite the LiI $4 p$ level from the ground state (see Figure 2). Collisional excitations and deexcitations by the plasma electrons lead to a rise in the populations of the $4 d$ and $4 f$ levels (and other neighboring levels) as shown by the time-dependent collisionalradiative calculations. The rise of the $3 d$ level population, for example, allows for measuring reliably the $2 p-3 d$ profile, which is used to determine the lithium velocity distribution, see below. Furthermore, because of the small energy separation between the $4 d$ and $4 f$ levels, the $4 d-4 f$ collisional excitation and de-excitation, for the plasma parameters used in this experiment, dominate other processes that may affect the $4 d$ and $4 f$ level populations. Collisional-radiative calculations thus show that the populations of these two levels (divided by the respective degeneracies) are equal to within $4 \%$, which is important for proper interpretation of the relative line intensities.

Li I

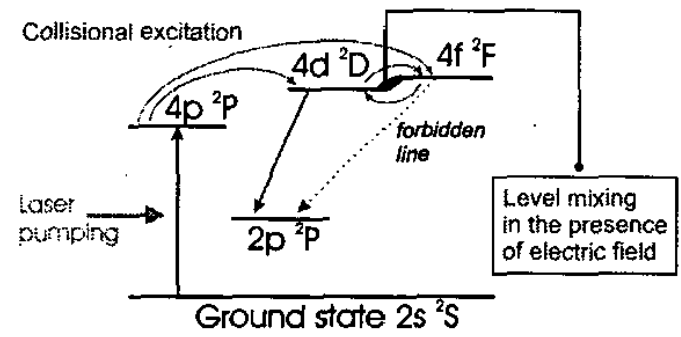

Figure 2. A diagram of the laser-driven excitation of the $\mathrm{LiI}$ levels.

Since the forbidden line amplitude strongly depends on the electric field strength [9], the forbidden line intensity is a reliable measure of the electric fields in the plasma. However, the determination of the forbidden-line intensity requires correct line-shape calculations for both the allowed and forbidden transitions, as is shown below.

\section{IV.RESULTS}

The technique developed was first applied for the determination of the properties of the laser-produced lithium beam, which is important for examining the effect of the dopant on the flashboard plasma conditions. Figure 3 gives the line-shape analysis. The instrumental response function of the spectroscopic diagnostic system was obtained from measurements of line spectra of $\mathrm{Ne}$ and $\mathrm{Hg}$ calibration lamps. The Doppler broadening was obtained from the spectral profile of the LiI $2 p-3 d$ transition (which is excited by collisional de-excitation of the $n=4$ level) by deconvolving the instrumental function from the total line shape (the $2 p-3 d$ transition is insensitive to Stark broadening and, for our plasma, its width is only affected by the Doppler broadening).

To analyze the $2 p-4 d$ spectral profile, we calculated the Stark-broadened spectrum for various values of the electron density $n_{e}$, and convolved it with the instrumental and the Doppler broadening functions. The electron density was determined by the best fit to the observed profile. For the calculations we used values of $2 \mathrm{eV}$ and $0.5 \mathrm{eV}$ for the ion $\left(T_{i}\right)$ and electron $\left(T_{e}\right)$ temperatures respectively. $T_{i}$ was obtained from the Doppler-dominated 
profile of the $2 p-3 d$ line as discussed above. The electron temperature is determined using time-dependent

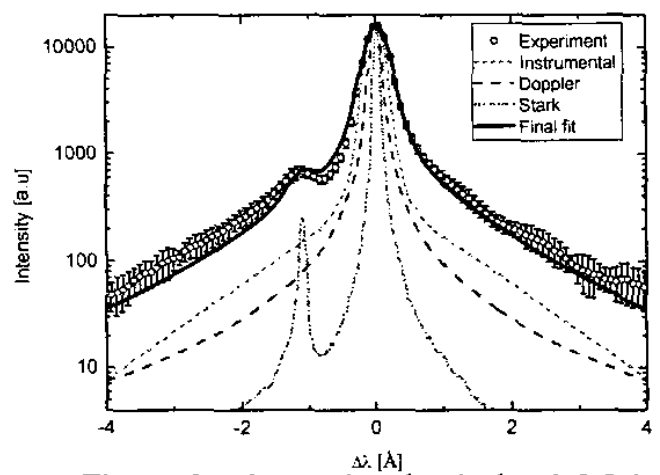

Figure 3. Measured and calculated LiI $2 p-4 d$ line shapes including the $2 p-4 f$ forbidden line at $2.5 \mathrm{~mm}$ from the cathode. The best fit shown is obtained with $n_{\mathfrak{c}}=4 \times 10^{13} \mathrm{~cm}^{-3}$.

collisional-radiative modeling of the temporal behavior of the intensities of the observed excited transitions (see below).

In the Stark-broadening calculations shown in Figure 3, the LS coupling was neglected since the fine splittings of the $2 p-4 d$ and $2 p-4 f$ lines are significantly smaller than the sum of the instrumental and the Doppler broadenings. From such calculations it was found that $n_{e}=(4 \pm 0.7) \times 10^{13} \mathrm{~cm}^{-3}$ provides a good fit to the spectra

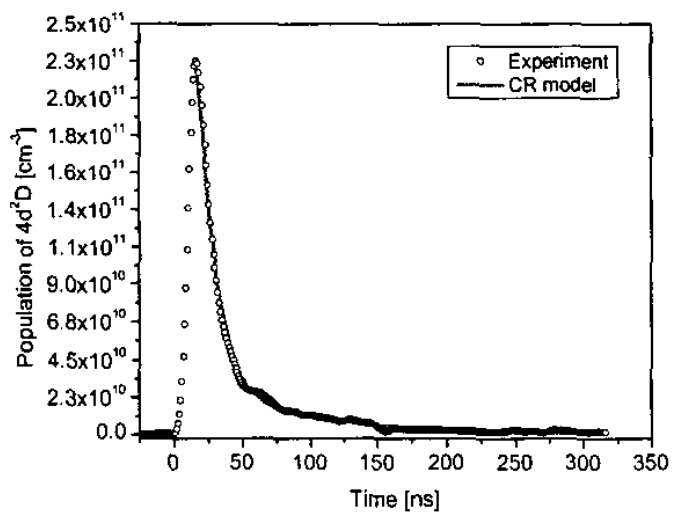

Figure 4. The LiI $4 d$-level population as a function of time following the application of the pumping laser pulse. The measurement was performed without the plasma prefill (only using the dopant lithium beam).

measured at $2.5 \mathrm{~mm}$ from the cathode, as shown in Figure 3.

The plasma electron density can also be determined from measurements and analysis of the temporal evolution of the LiI line intensities, following the photopumping of the $4 p$ level. To this end, detailed collisional-radiative calculations are used to model the populations of the $4 s, 4 p, 4 d, 4 f$, and $3 d$ levels, as shown by the example given in Figure 4 . The fit of the modeling results to all line intensities observed yield a reliable determination of the electron density and temperature.

The temporal behavior of several spectral line intensities $(2 p-4 s, 4972 A ; 2 s-4 p, 2741 A ; 2 p-4 d, 4603 A$; $2 p-3 d, 6104 A ; 2 s-2 p, 6708 A)$, emitted following the laser excitation, measured using the absolutely calibrated spectroscopic-PMT-array system, was thus inputted into collisional-radiative calculations $[10,7]$. The absolute line intensities and their time dependence are both self consistently modeled in order to determine the electron density and temperature. It was found that $n_{\mathrm{c}}=4 \times 10^{13} \mathrm{~cm}^{-3}$ and $T_{e}=0.5 \mathrm{eV}$ provide the best fit to the observed behavior of the level populations. The value of electron density found is in agreement with the result of the lineshape calculations described above, which further supports the validity of the two methods.

The method based on the line-shape analysis was then employed for experiments with the plasma prefill and the dopant lithium beam (no generator current was applied). Due to the flashboard-plasma injection the intensities of the LiI lines drop by $\cong 8 X$ (in comparison to the case of only lithium beam), because of the lithium ionization by

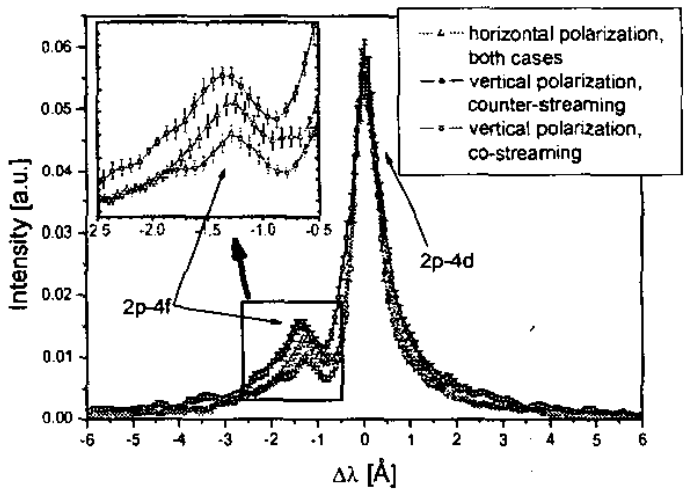

Figure 5. The LiI $2 p-4 d$ and $2 p-4 f$ lineshapes measured for two orthogonal polarizations for the two directions of the dopant injection.

the flashboard-plasma electrons (the flashboard plasma is of $n_{e}=2 \times 10^{14} \mathrm{~cm}^{-3}$ and $\left.T_{e} \sim 5 \mathrm{eV}[6]\right)$. However, the forbidden line has substantially higher relative intensity, which is shown by our line-shape calculations to result from the higher microfields in the higher-density flashboard plasma (the electron density in the lithium dopant is lower, $4 \times 10^{13} \mathrm{~cm}^{-3}$ as said above).

In the present study, the dopant lithium beam was injected in the same direction of the flashboard-plasma injection and opposite to it. In these measurements, in addition to the diagnostics described above, polarization spectroscopy was implemented, i.e., profiles of the lines were obtained for different polarizations.

The intensity of the forbidden $2 p-4 f$ line for the polarization parallel to the laser beam that produces the dopant (see Figure 1) was found to be similar for the two 
directions of the dopant injection. However, for the polarization parallel to the laser beam used for the optical pumping, the forbidden line intensity was found to be significantly higher for the dopant-injection direction opposite to the flashboard plasma, as shown in Figure 5. An analysis of this phenomenon will be reported on separately.

We now describe measurements during the application of the generator current. Here we use of the forbidden to allowed line ratios for determining the E-field in the plasma in the vicinity of the cathode surface (within $1 \mathrm{~mm}$ ) during the current conduction.

Figure 6 presents the forbidden-to-allowed line ratio as a function of time, where the current driven through the plasma starts at $t=0 \mathrm{~ns}$. Also shown in Figure 6 is the downstream current that rises after the current through the

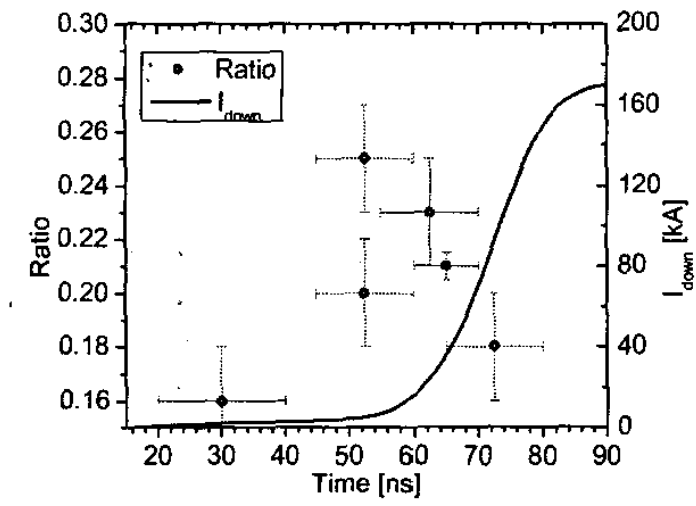

Figure 6. The ratio of the $L i I$ forbidden $(2 p-4 f)$ to the allowed $(2 p-4 d)$ line intensities during the current conduction. Here, $t=0$ is the beginning of the generator current pulse. Also shown is the downstream current (the current to the load) measured by a Rogowsky coil positioned at the load.

plasma decays (i.e., after the current switches to the load). It is seen in Figure 6 that the ratio prior to the rise of the generator current is $\cong 0.16$, which is consistent with $4 d-4 f$ mixing that results from the microfields in the $2 \times 10^{14}$ $\mathrm{Cm}^{-3}$ - density flashboard plasma discussed above. During the current conduction the ratio rises and reaches its peak value, with an average of 0.22 , at $\sim 55 n s$, when the entire generator current is still conducted by the plasma (very small fraction of the current flows to the load).

The reason for the rise of the forbidden-to-allowedline ratio requires further investigation. The ratio could rise due to a local rise in the plasma density, for example, due to plasma pushing by the magnetic field upstream of the measurement position. It can also be explained by a rise of electric fields in the plasma due to the current conduction. The electric field amplitude consistent with this ratio is $\sim 4 \mathrm{kV} / \mathrm{cm}$. If this field is associated with the Hall potential in the plasma, it can then be estimated from the term $\frac{B^{2}}{8 \pi n_{e} e}$, where $B$ is the value of the self magnetic field at the location of the measurement and $n_{e}$ is the local electron density. Using the typical values in our experiment for $B(1 \mathrm{~T})$ and for $n_{e}\left(2 \times 10^{14} \mathrm{~cm}^{-3}\right)$, one obtains a value of $12 \mathrm{kV}$. For such a potential to produce $E \simeq 4 \mathrm{kV} / \mathrm{cm}$, it must drop over a few $\mathrm{cm}$ (which should then be the current-channel width).

We believe that the diagnostic methods here described are applicable to a variety of transient plasmas under high currents.

\section{ACKNOWLEDGEMENTS}

We are highly grateful to H.R. Griem for fruitful discussions and critical comments on the line-shape analysis and to H.-J. Kunze for providing us with valuable help in the LIF experiments.

This work was supported in part by the Minerva Foundation, Munich (Germany), by the Israel Science Foundation, and by Sandia National Laboratories (USA).

\section{REFERENCES}

[1] Plasma diagnostics, edited by W. Lochte-Holtgreven New York : AIP Press, 1995.

[2] High-Resolution Laser Spectroscopy, edited by K. Shimoda. Springer, New York, 1976.

[3] W. Demtröder, Laser Spectroscopy, $2^{\text {nd }}$ edition, Springer, New York, 1996.

[4] E. Stambulchik, "Calculations of Spectral Line Broadening in Plasma", $\mathrm{PhD}$ thesis, Weizmann Institute of Science, 2002.

[5] R. Shpitalnik, A. Weingarten, K. Gomberoff, Ya. E. Krasik and Y. Maron, "Observations of twodimensional magnetic field evolution in a Plasma Opening Switch", Phys. of Plasmas 5(3). 792, 1998.

[6] A. Weingarten, V. Bernshtam, A. Fruchtman, C. Grabowski, Y. Krasik, and Y. Maron, "Study of the effects of the prefilled plasma parameters on the operation of a short-conduction plasma opening switch" IEEE Transaction on Plasma Science, 27, 1596 (1999).

[7] R. Arad, K. Tsigutkin, Yu.V. Ralchenko, and Y. Maron, "Spectroscopic investigations of a dielectricsurface-discharge plasma source", Physics of Plasmas 7. 3797, 2000.

[8] M. Baranger and B. Mozer, "Light as a plasma probe" Phys. Rev. 123 (1). 25, 1961.

[9] U. Rebhan, N.J. Wiegart and H.-J. Kunze, "Measurements of fluctuating electric fields by means of high-frequency stark effect in a laser excited lithium beam" Phys. Lett. 85A. 228, 1981.

[10] Yu.V. Ralchenko and Y. Maron, "Accelerated recombination due to resonant deexcitation of metastable states", J. of Quantitative Spect. and Rad. Transfer 71, 609, 2001. 